\title{
Investigating student understanding of basic quantum mechanics in the context of time-dependent perturbation theory
}

\author{
Gina Passante, Paul J. Emigh, and Peter S. Shaffer \\ Department of Physics, University of Washington, Seattle, WA 98195-1560
}

\begin{abstract}
Recent research suggests that many students complete an undergraduate course on quantum mechanics without having developed a solid understanding of some important fundamental concepts. We are probing the ability of students to apply foundational ideas in quantum mechanics in a variety of contexts, including the complex problem of time-dependent perturbation theory. Based on student responses to lecture post-tests, tutorial pretests and post-tests, as well as individual student interviews, we are identifying persistent difficulties that students have in applying basic ideas about energy measurements and the time evolution of both energy eigenstates and superpositions of energy eigenstates. The results are guiding the creation and revision of tutorials on topics students typically encounter in the second half of their undergraduate instruction on quantum mechanics.
\end{abstract}

Keywords: physics education research, student understanding, quantum mechanics, perturbation theory, upper division PACS: 01.40.G-, 03.65.Ta, 03.65.-w

\section{INTRODUCTION}

Quantum mechanics $(\mathrm{QM})$ is one of the capstone courses in a physics degree, but the topic is becoming increasingly relevant for studies in other fields such as engineering, biology, and chemistry. It is a very difficult subject for students to learn; not only are the predictions counter to everyday experiences and prior instruction in classical physics, but the topic is also typically presented using a new mathematical formalism. Previous research confirms these assertions [1-5] and indicates that most students struggle with similar concepts, regardless of instructor, textbook, or institution; a situation that is not unlike that at the introductory level [6].

The Physics Education Group at the University of Washington (UW) is developing a set of Tutorials in Physics [7] for upper-division QM. These follow the tradition of Tutorials in Introductory Physics [8], with the goal of improving student understanding of QM at a conceptual level.

In this work we report on an investigation into student reasoning of basic, foundational ideas within the complex context of time-dependent perturbation theory (TDPT). Here, we define foundational concepts as those that are taught early in a QM course and that are required for a full understanding of TDPT. In this context, two foundational ideas of interest are (1) the time dependence of quantum states and (2) energy measurements. Previous research indicates that many students at all levels of instruction on QM, including incoming graduate students, have difficulty with these concepts.

Regarding (1) the time dependence of quantum states, it is important to understand that energy eigenstates are stationary, whereas superpositions of (non-degenerate) energy eigenstates have a spatial probability density that depends on time. Previous research, from Singh for example [3], found that about one-third of incoming graduate students incorrectly wrote a common time-dependent phase factor superpositions of energy eigenstates, which would result in probability density that does not depend on time. Additionally, about $10 \%$ of students believe that there are states without any time dependence.

With regard to (2) energy measurements, it is important for students to understand that an energy measurement can only yield the eigenvalues of the Hamiltonian, which is dependent on the potential. Previous work by Crouse [5] reports that only twothirds of graduate students could correctly identify the possible results of an energy measurement and their associated probabilities. It is clear that these ideas are difficult for even the best students, and persist well after initial instruction.

We had designed tutorials for early in the study of QM to address these and other difficulties with time dependence and energy measurements. However, during the development of more advanced tutorials near the end of the sequence, it came to our attention that students were still struggling with basic ideas that had been specifically addressed in previous tutorials. 
A time dependent perturbation to the infinite square well

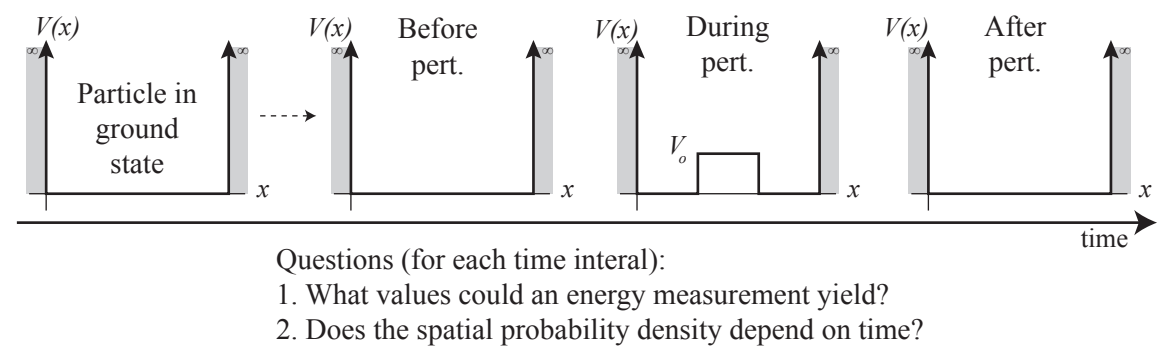

FIGURE 1. This diagram depicts the pretest question students were given in 2005-07 where a small perturbation is applied to an infinite square well. Students were asked to only use first order perturbation theory when answering the questions and they were told that the potential of the perturbation was much smaller than the ground state energy.

This manuscript focuses on identifying some of the specific difficulties students encounter with these foundational ideas when confronted with a situation involving TDPT. In the rest of this paper, we report student facility with these foundational ideas after tutorial instruction specifically designed to address them. We give results from pre-tests administered immediately before a TDPT tutorial (but after TDPT lecture instruction), followed by a description of the tutorial and post-test data. Finally, we present findings from individual student interviews after all instruction on QM. We conclude with a discussion of how this information is informing ongoing development of curricular materials not only for TDPT, but also that better target these foundational ideas during earlier instruction.

\section{CONTEXT FOR RESEARCH}

This research has taken place in the context of the two-quarter junior-level QM sequence at the UW. These courses use David Griffith's Introduction to Quantum Mechanics [9] textbook, covering the entire book over two quarters. The foundational ideas of interest are taught in the beginning of the first quarter, while TDPT falls near the end of the second quarter. The courses meet for three 50-minute lectures each week, as well as one 50-minute small group section per week. The lectures were taught in a traditional (noninteractive) manner. During some weeks, the small group sections used tutorials; in other weeks they consisted of problem solving sessions led by a graduate teaching assistant. Students in these courses have also completed a one-quarter course at the sophomore level on modern and quantum physics, which discusses some of the foundational ideas mentioned above.

\section{Foundational Tutorial Post-tests}

We have given a variety of post-tests to assess the extent to which the early tutorials address student difficulties with the foundational concepts described above. In the case of energy measurements, about $65 \%$ of the students after the relevant tutorials seem to recognize that the energy eigenvalues depend on the particular form of the potential. Likewise, about $75 \%$ of the students recognize that the probability density is constant for energy eigenstates but changes with time for a (non-degenerate) superposition of energy eigenstates.

\section{TDPT Tutorial Pretest}

A number of different tutorial pretests on TDPT have been administered after lecture instruction on this topic and after the tutorials on foundational topics, but before the TDPT tutorial. Data presented below was collected from 2005-07. The pretest was based on a typical TDPT scenario in which an infinite square well is perturbed for a given time interval and then returned to its original form, as illustrated in Fig. 1. Students were told that a long time before the perturbation is applied, the system was measured to be in the ground state. For each time interval, students were asked for the possible results of an energy measurement and whether or not the spatial probability density depends on time.

Energy measurements: The results from the energy measurement question (question 1 in Fig. 1) are given in Table 1.

TABLE 1. Percentage of correct responses

\begin{tabular}{c|c|c|c}
\hline & Before pert. & During pert. & After pert. \\
\hline $2005-07(71)$ & $50 \%(35)$ & $55 \%(39)$ & $15 \%(12)$ \\
\hline
\end{tabular}

Many students stated that because the perturbation had not yet been applied, all energies are possible for the time before the perturbation. They failed to recognize that since the system was in the ground state initially, it would be in the ground until the perturbation began. For times during the perturbation, some students incorrectly answered that a "linear combination of [the unperturbed] energies" would be 
possible. Others seemed to think that the perturbation 'added' energy to the system and tried to calculate a range of possible energies by adding the ground state energy to the 'perturbation energy $\left(V_{o}\right)$ '. For the time period after the perturbation, the most common incorrect response was that only the ground state energy was possible. Many students seemed to think that the state would return to what it was prior to the perturbation.

Time dependence of probability density: On the second question regarding the spatial probability density (question 2 in Fig. 1), only 25\%, 15\%, and $15 \%$ of the students were able to answer correctly with correct reasoning for the time period before, during, and after the perturbation, respectively. The most common incorrect reasoning for the time periods before and after the perturbation was that the wave function is time-independent and thus, so is the probability density. Another common incorrect explanation was that since the Hamiltonian is time independent, the probability would be as well. During the perturbation, the most common incorrect reasoning was similar. Many students stated that the perturbation caused the Hamiltonian to be time-dependent, giving rise to a time-dependent probability density.

\section{TDPT Tutorial Instruction}

Two distinct versions of the tutorial have been administered. From 2005-07 the tutorial was designed to walk students through the derivation of the equation for TDPT in addition to addressing some of the foundational ideas identified in the pretest as difficult for students. In 2013 the tutorial was modified to have less of a focus on the derivation and greater attention was given to having students examine the context and reflect on how the equation fits with this context.

\section{TDPT Tutorial Post-test}

Results presented here are from post-test questions on TDPT from final exams in 2006 and 2013. Both questions involved a perturbation applied to a harmonic oscillator potential that was initially in the first excited state (which has energy $E_{1}$ ).

In 2006, students $(N=41)$ were asked to rank the probability of measuring $E_{0}$ (and subsequently $E_{2}$ and $\left.E_{3}\right)$ before, during, after, and a long time after the perturbation. They were asked to specify explicitly if any of the probabilities were equal to zero or one. This question is more difficult than the pretest questions, in part due to the fact that students are asked to rank the probabilities. In addition, the potential and the perturbation were more complex. This extra difficulty was evident in student responses. Only 15\%, 25\%, and
$25 \%$ of the students correctly indicated that the probability of measuring $E_{0}, E_{2}$, and $E_{3}$, respectively, during the perturbation is zero. In order to answer this correctly, students needed to recognize that these energies are not eigenvalues of the Hamiltonian while the perturbation is turned on.

On the 2013 final exam, students $(N=48)$ were asked to determine the probability of measuring the energy value $E_{1}$ while the perturbation is turned on. Students did fairly well, with $60 \%$ of them correctly stating that the probability is zero. Most of the students who responded incorrectly treated the probability as depending on time. The question also asked for the probability that $E_{0}$ is measured after the perturbation is turned off. About 40\% correctly calculated this probability to be non-zero, while $35 \%$ indicated that $E_{0}$ could not be measured. Most of the incorrect answers stated that after the perturbation was removed, the wave function returns to its state prior to the perturbation (which in this case was $\psi_{1}$ ). Note that this is the same incorrect reasoning as seen on pretests.

\section{INTERVIEWS}

In order to gain greater insight into how students are thinking about these questions, we performed four student interviews in the spring quarter of 2013. These were conducted after the conclusion of the second quarter quantum mechanics course. All students interviewed had passed both courses and attended all tutorial sessions.

In the interview, students were shown a timeline that outlined a sequence of events for a quantum particle. The particle began in a superposition of two (non-degenerate) energy eigenstates. Then, an energy measurement was made and students were informed of the outcome. The potential in which the particle was bound was then quickly changed, and some time later it was quickly returned to its original form. The functional form for the potential, perturbation, or energy eigenstates was intentionally not given. This encouraged students to think more generally about the scenario and not rely on mathematics alone. The students were asked about possible results of energy measurements in each time interval, as well as the possible time dependence of the probability density and wave function.

After these questions were answered, interviewees were asked more straightforward questions about the time dependence and energy measurements of a particle in an infinite square well. They were then asked to revisit the TDPT questions. We discuss the interviews of two students that are indicative of the results mentioned above.

The first student had received a 4.0 grade point average in both junior-level QM courses. He did not 
have a problem in correctly answering the questions about the possible results of an energy measurement, but incorrectly reasoned about the time dependence. Originally he indicated that neither the probability density nor the wave function of the particle had any time dependence at any time except during the perturbation. He reasoned that: "...the coefficient for probability of these eigenstates [during the perturbation time frame] have a time dependence and this one [the initial superposition state] doesn't." $\mathrm{He}$ was then presented with the infinite square well scenario and asked the same questions. In this context he almost immediately recognized that both the wave function and the spatial probability density of a superposition of infinite square well energy eigenstates would have time dependence. When the interview returned to the TDPT scenario he immediately said "Okay. I think some of my answers will change," and proceeded to correct his previous mistakes.

The second interviewee was in the top $20 \%$ in both classes. She correctly answered the energy questions for the time periods before the perturbation, but was unable to reason correctly about what happened during or after the perturbation. She correctly answered the questions about energy measurement for the infinite square well scenario, but did not revise her answers when revisiting the TDPT scenario.

Like the first interviewee, she also had difficulty answering the questions on time dependence. She indicated that the wave function did not any have time dependence, except during the perturbation. When asked if she had a rule to help her determine when there is time dependence, her response was: "See, I only know if it's written explicitly. I feel like you can write it and it won't change anything. Well, it will change something, obviously. But, I feel like in my head I see this sum with the eigenvectors with that [exponential term] tacked on at the end to add time dependence. But here I don't see any time dependence." When confronted with the infinite square well problem, she had some internal debate, and then decided that the infinite square well wave function needs the time dependence, and added it correctly. When revisiting the TDPT scenario she correctly changed her answers to indicate that the state depends on time everywhere, and the superposition states have a probability density that depends on time.

\section{DISCUSSION}

During our investigation of student understanding of TPDT, we found that students were struggling with seemingly simple questions about foundational ideas. These ideas had been explicitly addressed in previous tutorial curricula, and an improvement in student understanding was observed. However, it appears that within the more difficult context of TDPT, students failed to apply the information they had previously learned.

TDPT tutorial pretests indicate that students perform worse on these foundational questions in the TDPT context than on more straightforward questions immediately after prior tutorial instruction. While the TDPT tutorial briefly addresses these ideas, post-tests show that it was not effective enough at assisting students in transferring the foundational ideas to this complex context.

Individual student interviews with top students show that even students performing near at the top of the class struggle with these ideas, which is consistent with previous research $[3,5]$. These difficulties are proving to be persistent and are hindering some students' ability to master more difficult, and often more physically relevant, topics. Further research into student ideas about these topics is required in order to improve the preparation of students for further study in physics, or for applying QM to other areas such as engineering, biology, and chemistry. We are working to develop instructional strategies that will deepen student understanding of both basic and advanced quantum mechanical ideas.

\section{ACKNOWLEDGMENTS}

The authors would like to thank current and past members of the Physics Education Group at the University of Washington, in particular Andrew Crouse, who designed the original TDPT tutorial. We would also like to thank UW faculty members Andreas Karch and John Rehr for welcoming this research in their courses. This research has been supported in part by the National Science Foundation under Grant No. DUE-1022449.

\section{REFERENCES}

1. C. Singh, Am. J. Phys. 69, 885 (2001).

2. G. Zhu and C. Singh, Am. J. Phys. 80, 252 (2012).

3. C. Singh, American Journal of Physics 76, 277 (2008).

4. D.F. Styer, Am. J. Phys. 64, 31 (1996).

5. A. Crouse, Student Understanding of Quantum Mechanics, University of Washington, 2007.

6. L.C. McDermott and E.F. Redish, American Journal of Physics 67, 755 (1999).

7. L.C. McDermott, P.S. Shaffer, P.R.L. Heron, and the Physics Education Group, Tutorials in Physics, Preliminary edition (2013).

8. L.C. McDermott, P.S. Shaffer, the Physics Education Group, Tutorials in Introductory Physics (Prentice Hall College Div, 2001).

9. D.J. Griffiths, Introduction to Quantum Mechanics, 2nd ed. (Pearson Prentice Hall, Upper Saddle Rive, NJ, 2005). 